\title{
Prevalence of Risk of Malnutrition and Risk of Sarcopenia in a Reference Hospital for COVID-19: Relationship with Mortality
}

\author{
Henar Riesgo Angeles Castro Sofía del Amo Maria Jesus San Ceferino \\ Olatz Izaola David Primo Emilia Gómez Hoyos Juan Jose López Gómez \\ Daniel A. de Luis \\ Center of Investigation of Endocrinology and Nutrition, Medicine School, Department of Endocrinology and \\ Nutrition, Hospital Clinico Universitario, University of Valladolid, Valladolid, Spain
}

\section{Keywords}

COVID-19 · Mortality · Malnutrition · Sarcopenia

\begin{abstract}
Introduction: Many elderly patients with COVID-19 are at risk of malnutrition. The aim of our study was to evaluate the risk of malnutrition and sarcopenia in elderly COVID-19 patients with the R-MAPP (Remote-Malnutrition APP). Materials and Methods: A cross-sectional study of 337 consecutive outpatients $\geq 65$ years who attended the Central Emergency COVID-19 Hospital of Castilla y Leon was conducted. In all patients, the protocol of R-MAPP (Malnutrition Universal Screening Tool [MUST] and Simple Questionnaire to Rapidly Diagnose Sarcopenia [SARC-F]) was realized. Results: The mean age was $86.1 \pm 8.7$ years, with a sex distribution of 167 males (49.5\%) and 170 females (51.5\%). According to the MUST test, patients with 0 points have a low nutritional risk ( $n=50,14.8 \%), 1$ point a medium nutritional risk $(n=19$, $5.6 \%)$, and 2 or more points a high nutritional risk ( $n=268$, $79.6 \%$ ). The SARC-F questionnaire generates patients with 4 or more points as predictive of sarcopenia ( $n=304,80.2 \%$ ) and $<4$ points without prediction of sarcopenia $(n=33$, $9.8 \%)$. Global mortality was $24.03 \%(n=81)$. The mortality
\end{abstract}

rate was related to the pathological SARC-F score $\geq 4(27.1 \%$ vs. $3.1 \% ; p=0.01)$ and MUST score $\geq 2$ (26.7\% vs. $16.4 \% ; p=$ $0.04)$. In the logistic regression analysis, only the SARC-F score $\geq 4$ remained as an independent variable related to mortality; odds ratio was 8.34 (95\% Cl: 1.1-63.8; $p=0.04$ ), adjusted for age, sex, albumin levels, and MUST test. Conclusions: During COVID-19 infection, hospitalized patients at risk of sarcopenia have a high risk of mortality and have a poor nutritional status.

(c) 2021 S. Karger AG, Basel

\section{Introduction}

Since the declaration of the coronavirus disease-2019 (COVID-19) pandemic on March 11, 2020, this disease has spread across the world, changing everyday life and impacting the healthcare system. COVID-19 is an infectious disease caused by the coronavirus SARS-CoV-2. COVID-19 produces a respiratory infection characterized by mild to severe symptoms [1].

Clinical observations have detected that many elderly patients with COVID-19 are at risk of malnutrition. In addition, SARS-CoV-2 can damage the mucosal epithe- karger@karger.com

(c) 2021 S. Karger AG, Basel

www.karger.com/anm

Karger ${ }^{\prime \prime}=$
Correspondence to:

Daniel A. de Luis, dadluis@yahoo.es 
lium and cause gastrointestinal changes, which affect the nutritional status of these patients [2]. Additionally, most of the countries have seen their health systems overwhelmed, especially the capacity of their hospitals. For this reason, in some areas, central emergency hospitals have been opened to care for elderly patients infected with COVID-19, with few health personnel specialized in nutritional assessment. This situation has made it necessary to promote telemedicine measures from nutrition units to give assistance to these new hospitals. Telemedicine is considered as a useful remote communication and information technology to provide medical services to patients in different locations. With these new circumstances, it is crucial to endorse pragmatic protocols for the nutritional assessment of nutritional support in these emergency hospitals, and recently a simple remote nutritional screening tool has been developed by the European Society of Parenteral and Enteral Nutrition (ESPEN) [3]. This platform proposes the use of 2 validated clinical tools to search nutritional risk with the Malnutrition Universal Screening Tool (MUST) [4] and the Simple Questionnaire to Rapidly Diagnose Sarcopenia (SARC-F) as a test to predict sarcopenia [5]. The acronym for the tool is RMAPP, as for Remote-Malnutrition APP, and the platform was developed as an app tool. The aim of our study was to evaluate the risk of malnutrition and sarcopenia in elderly COVID-19 patients with the R-MAPP tool in a central emergency COVID-19 hospital of our country.

\section{Research Design and Methods}

A cross-sectional study of 337 consecutive hospitalized patients $\geq 65$ years who attended the Central Emergency COVID-19 Hospital of Castilla y Leon between November 2020 and March 2021 was conducted. All participants gave written informed consent to the protocol approved by the local ethical review boards. This study was realized with the guidelines laid down in the Declaration of Helsinki. The local clinical research ethics committee (PI20-1776) approved this study.

All patients were confirmed with COVID-19 infection by real-time RT-PCR with the following criteria of inclusion: age over 65 years, hospitalized with a length of stay $>24$ h, stable clinical situation characterized by respiratory rate $<20$ breaths per minute, absence of any type of ventilatory support, hemodynamic stability, normal level of consciousness, absence of other poor prognostic criteria (lymphopenia $<1,200 / \mathrm{mm}^{3}$, D-dimer $>1,500 \mathrm{ng} / \mathrm{mL}$, and increasing ferritin), and finally, patients without care criteria in a critical care unit since the central emergency hospital for COVID-19 did not have these facilities, and it was one of the criteria for hospital admission. Exclusion criteria were as follows: age $<65$ years, severe renal dysfunction, severe liver dysfunction, and patients unable to get anthropometric parameters.
Table 1. Basal characteristics of the patients

\begin{tabular}{ll}
\hline Parameters (total group, $n=337$ ) & Basal data \\
\hline Age, years & $86.1 \pm 8.7$ \\
BMl & $23.8 \pm 2.8$ \\
Weight, $\mathrm{kg}$ & $62.1 \pm 10.3$ \\
Hemoglobin, $\mathrm{g} / \mathrm{dL}$ & $12.4 \pm 1.8$ \\
Leucocytes, $\times 10^{9} / \mathrm{L}$ & $8.6 \pm 4.4$ \\
Platelets, $\times 10^{9} / \mathrm{L}$ & $263.1 \pm 125.1$ \\
Fasting glucose, $\mathrm{mg} / \mathrm{dL}$ & $103.1 \pm 35.9$ \\
Total cholesterol, $\mathrm{mg} / \mathrm{dL}$ & $156.5 \pm 31.8$ \\
Triglycerides, $\mathrm{mg} / \mathrm{dL}$ & $143.9 \pm 52.9$ \\
Creatinine, $\mathrm{mg} / \mathrm{dL}$ & $1.1 \pm 0.6$ \\
Sodium, $\mathrm{mEq} / \mathrm{L}$ & $140.5 \pm 4.5$ \\
Potassium, $\mathrm{mEq} / \mathrm{L}$ & $4.5 \pm 1.0$ \\
Albumin, $\mathrm{g} / \mathrm{dL}$ & $3.3 \pm 0.3$ \\
Prealbumin, $\mathrm{mg} / \mathrm{dL}$ & $18.6 \pm 6.6$ \\
Hospital stay, days & $11.1 \pm 6.7$ \\
\hline
\end{tabular}

Basal data $<24 \mathrm{~h}$ after hospital admission. BMl, body mass index.

\section{Demographics, Nutritional Status, and Procedures}

Demographic characteristics, such as age and sex, were recorded from the electronic medical record. The comorbidities were determined by patients' reports from medical history. During the first $24 \mathrm{~h}$ of hospitalization, the protocol of R-MAPP (with MUST and SARC-F tests) was realized from our Nutrition Unit to the hospitalization areas of this central emergency hospital with the nursing teams of those hospitalization areas. Three criteria were used in "MUST" to assess the risk of malnutrition: BMI, unintentional weight loss, and acute disease effect [6]. In order to assess loss of muscle mass and function without any diagnostic tools and procedures, the SARC-F questionnaire can be used as a rapid diagnostic test for sarcopenia based only on muscle contractile performance items: strength, assistance with walking, rise from a chair, climb stairs, and falls [7]. On the first day of hospital admission, biochemical parameters were measured in a fasting situation of $10 \mathrm{~h}$.

The type of oral diet prescribed by a responsible physician of the patient was recorded. Nutritional supplementation with oral nutrition supplement secondary to the assessment with the RMAPP, in order to achieve the objective requirements in these patients $(30 \mathrm{kcal}$ per $\mathrm{kg}$ crude body weight per day and $1 \mathrm{~g}$ protein per kg crude body weight per day) [8], was also recorded.

\section{Biochemical Analysis}

Hemoglobin, leukocytes, and platelets of the patients were determined in a total blood sample, and they were processed and analyzed with XN 1000 (Sysmex, Madrid, Spain). Biochemical parameters such as glucose, creatinine, sodium, potassium, albumin, prealbumin, total cholesterol, and triglycerides were measured with the c501 module of the COBAS C 6000 device (Roche Diagnostics S.L., Sant Cugat del Vallès, Spain).

The diagnosis of COVID-19 was carried out by using real-time RT-PCR assay (LightMIx Modular SARS-CoV [COVID-19] Egene; Roche Diagnostics S.L.). Previously, SARS-CoV-2 RNA was isolated from an oropharyngeal swab using the MagnaPure compact RNA Isolation kit (Roche Diagnostics S.L.). 
Table 2. Biochemical and anthropometric parameters: MUST 0-1 point versus MUST $\geq 2$ points

\begin{tabular}{llll}
\hline & MUST 0 or 1 point & MUST $\geq 2$ & $p$ value \\
\hline Age, years & $84.6 \pm 9.1$ & $86.3 \pm 8.1$ & 0.33 \\
BMl & $23.4 \pm 2.7$ & $24.0 \pm 2.8$ & 0.41 \\
Weight, $\mathrm{kg}$ & $59.0 \pm 10.5$ & $61.7 \pm 10.1$ & 0.38 \\
Hemoglobin, $\mathrm{g} / \mathrm{dL}$ & $12.5 \pm 1.7$ & $12.3 \pm 1.2$ & 0.29 \\
Leucocytes, $\times 10^{9} / \mathrm{L}$ & $8.1 \pm 4.0$ & $8.8 \pm 2.1$ & 0.21 \\
Platelets, $\times 10^{9} / \mathrm{L}$ & $239.1 \pm 125.1$ & $275.3 \pm 103.2$ & 0.48 \\
Fasting glucose, $\mathrm{mg} / \mathrm{dL}$ & $104.6 \pm 27.9$ & $104.3 \pm 23.9$ & 0.31 \\
Total cholesterol, $\mathrm{mg} / \mathrm{dL}$ & $155.9 \pm 31.8$ & $158.9 \pm 28.8$ & 0.33 \\
Triglycerides, $\mathrm{mg} / \mathrm{dL}$ & $141.0 \pm 44.9$ & $144.9 \pm 25.9$ & 0.14 \\
Creatinine, $\mathrm{mg} / \mathrm{dL}$ & $1.0 \pm 0.3$ & $1.1 \pm 0.6$ & 0.18 \\
Sodium, $\mathrm{mEq} / \mathrm{L}$ & $139.1 \pm 4.2$ & $141.7 \pm 4.1$ & 0.42 \\
Potassium, $\mathrm{mEq} / \mathrm{L}$ & $4.2 \pm 1.1$ & $4.6 \pm 1.3$ & 0.33 \\
Albumin, $\mathrm{g} / \mathrm{dL}$ & $3.26 \pm 0.2$ & $3.3 \pm 0.3$ & 0.22 \\
Prealbumin, $\mathrm{mg} / \mathrm{dL}$ & $18.2 \pm 3.8$ & $18.6 \pm 5.1$ & 0.39 \\
Hospital stay, days & $9.9 \pm 8.4$ & $10.3 \pm 6.4$ & 0.23 \\
\hline
\end{tabular}

Basal data $<24 \mathrm{~h}$ after hospital admission. No statistical differences. BMI, body mass index.

\section{Statistical Analysis}

The quantitative variables are expressed as means \pm SD and the qualitative ones according to their frequency distribution. By means of Pearson's $\chi^{2}$ test, the association of qualitative variables was analyzed. In the case that the number of cells with expected values $<5$ is $>20 \%$, Fisher's exact test or the likelihood ratio test has been used for variables with $>2$ categories. The comparisons of the quantitative values have been made using the Student's $t$ test or the ANOVA of a factor for independent samples according to whether the number of groups to be compared is 2 or more.

A multivariate logistic regression analysis was carried out to predict the risk of mortality. The variables that have been found to be statistically significant at level 0.1 have been included in this logistic analysis. The data have been analyzed with the statistical program IBM SPSS Statistics version 23.0 for Windows (Chicago, IL, USA). Those values of $p<0.05$ have been considered statistically significant.

\section{Results}

Three hundred and thirty-seven patients were enrolled. The mean age was $86.1 \pm 8.7$ years, with a sex distribution of 167 males (49.5\%) and 170 females (51.5\%). The most frequent comorbidities were hypertension (37.3\%), dementia (28.7\%), diabetes mellitus (14.7\%), chronic lung disease (6.1\%), mild chronic kidney disease $(4.5 \%)$, ischemic heart disease (3.8\%), digestive pathology $(2.9 \%)$, ictus $(2.5 \%)$, and anxiety and mental disease (2.5\%).

Table 1 shows biochemical and anthropometrical data. According to the MUST test, patients with 0 points have a low nutritional risk $(n=50,14.8 \%)$, patients with 1 point have a medium nutritional risk $(n=19,5.6 \%)$, and patients with 2 or more points have a high nutritional risk ( $n=268,79.6 \%)$. R-MAPP is classified into 2 groups: MUST 0 or 1 point $(n=59,20.4 \%)$ and MUST 2 or more points $(n=268,79.6 \%)$. The SARC-F questionnaire generates patients with 4 or more points as predictive of sarcopenia $(n=304,80.2 \%)$ and $<4$ points without prediction of sarcopenia $(n=33,9.8 \%)$. The 3 oral diets with the highest prescription were texture modification diet for dysphagia (48.5\%), 20.4\% diet with modifications for hypertension or diabetes, $11.9 \%$ diet with modifications for chewing problems, $8.0 \%$ easy digestion diet, and the remaining $11.2 \%$ were normal diet. A total of $40.8 \%$ of patients were receiving oral supplementation in the form of oral nutrition supplement in order to reach $30 \mathrm{kcal}$ per $\mathrm{kg}$ body weight per day and $1 \mathrm{~g}$ protein per $\mathrm{kg}$ body weight per day in older patients [8].

Table 2 shows biochemical and anthropometrical data in 2 different groups: MUST 0 or 1 point versus MUST 2 or more points. No anthropometric, biochemical parameter showed differences with these 2 groups of nutritional risk. The hospital stay did not show significant differences either.

Table 3 shows biochemical and anthropometrical data in 2 different groups: SARC-F with 4 or more points versus SARC-F $<4$ points. Age was higher in the group with SARC-F $\geq 4$ points. However, the body weight was lower. The remaining parameters had no differences between both groups. The hospital stay was similar too. 
Table 3. Biochemical and anthropometric parameters: $S A R C-F<3$ points versus $\mathrm{SARC}-\mathrm{F} \geq 4$ points

\begin{tabular}{llll}
\hline & SARC-F $(<3$ points $)$ & SARC-F $(\geq 4$ points $)$ & $p$ value \\
\hline Age, years & $79.2 \pm 12.1$ & $86.3 \pm 8.1$ & 0.03 \\
BMl & $24.6 \pm 3.1$ & $23.8 \pm 2.1$ & 0.11 \\
Weight, $\mathrm{kg}$ & $65.5 \pm 11.5$ & $61.6 \pm 10.0$ & 0.02 \\
Hemoglobin, $\mathrm{g} / \mathrm{dL}$ & $12.8 \pm 1.8$ & $12.3 \pm 1.4$ & 0.29 \\
Leucocytes, $\times 10^{9} / \mathrm{L}$ & $8.4 \pm 4.5$ & $8.6 \pm 2.4$ & 0.39 \\
Platelets, $\times 10^{9} / \mathrm{L}$ & $270.1 \pm 135.1$ & $259.3 \pm 143.2$ & 0.41 \\
Fasting glucose, $\mathrm{mg} / \mathrm{dL}$ & $96.1 \pm 31.9$ & $105.7 \pm 33.9$ & 0.33 \\
Total cholesterol, $\mathrm{mg} / \mathrm{dL}$ & $155.2 \pm 40.8$ & $158.2 \pm 28.8$ & 0.46 \\
Triglycerides, $\mathrm{mg} / \mathrm{dL}$ & $143.0 \pm 52.9$ & $143.9 \pm 35.9$ & 0.54 \\
Creatine, $\mathrm{mg} / \mathrm{dL}$ & $0.9 \pm 0.6$ & $1.1 \pm 0.7$ & 0.28 \\
Sodium, $\mathrm{mEq} / \mathrm{L}$ & $140.1 \pm 4.1$ & $140.7 \pm 4.8$ & 0.41 \\
Potassium, $\mathrm{mEq} / \mathrm{L}$ & $4.5 \pm 1.0$ & $4.6 \pm 1.2$ & 0.65 \\
Albumin, $\mathrm{g} / \mathrm{dL}$ & $3.6 \pm 0.2$ & $3.2 \pm 0.4$ & 0.22 \\
Prealbumin, $\mathrm{mg} / \mathrm{dL}$ & $17.7 \pm 6.8$ & $18.7 \pm 5.9$ & 0.37 \\
Hospital stay, days & $8.9 \pm 6.4$ & $10.4 \pm 6.9$ & 0.53 \\
\hline
\end{tabular}

Basal data $<24 \mathrm{~h}$ after hospital admission. No statistical differences. BMI, body mass index.
Global mortality was $24.03 \%(n=81)$. The mortality rate was related to the pathological SARC-F score $\geq 4$ $(27.1 \%$ vs. $3.1 \% ; p=0.01)$ and MUST score $\geq 2(26.7 \%$ vs. $16.4 \% ; p=0.04)$. The other qualitative variables, such as comorbidities, type of oral diet, diabetes mellitus, gender, and use of NOS, were not associated with mortality. In the univariate analysis of quantitative variables, there were only differences in age, which was higher in patients who died ( $85.1 \pm 9.3$ vs. $88.7 \pm 4.3$ years; $p=0.01)$, and in albumin levels, which was lower in patients who died (3.3 \pm 0.3 vs. $3.1 \pm 0.1 \mathrm{~g} / \mathrm{dL} ; p=0.01)$. In the logistic regression analysis, only the SARC-F score $\geq 4$ remained as an independent variable related to mortality; odds ratio was 8.34 (95\% CI: $1.1-63.8 ; p=0.04$ ), adjusted for age, sex, albumin levels, and MUST test.

\section{Discussion}

The results of this study show that elderly patients with COVID-19 who were classified as having a sarcopenia risk had a higher significant mortality than those classified as normal. The MUST showed a high prevalence of nutritional risk in these patients.

Elderly patients (over 65 years) have been related to worse prognosis and severe COVID-19 disease [9]. Factors such as the greater propensity for organ dysfunction, presence of comorbidities, and poor nutritional status can influence this risk of complications in elderly patients with COVID-19 [10]. COVID-19 disease presents with inflammatory condition characterized by the involvement of proinflammatory cytokines [11], and secondarily malnutrition may have worsened the patient's immune system and, consequently, the effectiveness of treatment, producing a greater severity of the disease [12]. Considering this scenario, the early assessment of nutritional risk can contribute to the determination of the appropriate nutritional therapy, better immune system response, and favorable prognosis [13]. In a recent review, Silva et al. [14] reported that the Nutritional Risk Screening 2002 (NRS-2002), the Mini Nutritional Assessment (MNA), the MNA-short form (MNA-sf), the Malnutrition Universal Screening Tool (MUST), the Nutritional Risk Index (NRI), the Geriatric NRI (GNRI), and modified Nutrition Risk in the Critically ill (mNUTRIC) score demonstrated high sensitivity to detect malnutrition in COVID-19 patients. The MUST test had better specificity with better criterion validity [14]. So, no consensus exists on the most appropriate method to identify nutritional risk in these patients, and bearing in mind that our health system was overwhelmed and lacking specialized personnel to carry out nutritional assessments, we decided to use the R-MAPP that included the MUST test. Our data show a high risk of malnutrition (MUST $>2$ ) of $79.6 \%$, similar to other research studies. For example, the prevalence rates of nutritional risk in Liu et al. [15] study were $85.1 \%$ (NRS-2002), 77.3\% (MNA-sf), 60.4\% (NRI), and $41.1 \%$ (MUST). Li et al. [16] found that $27.5 \%$ of patients were at risk of malnutrition and $52.7 \%$ were malnourished. Based on mNUTRIC scores, Zhang et al. [17] 
found that $61 \%$ of patients had a high nutritional risk, and finally Yuan et al. [18] reported a $100 \%$ of high nutritional risk with the GNRI test.

The mortality ratio in our study was high, as in other studies that have evaluated older patients with $\mathrm{CO}$ VID-19. In a previous Spanish registry, Carrasco et al. [19] reported a $20.4 \%$ in-hospital mortality ratio in noncritical patients hospitalized with COVID-19. Genc Yavuz et al. [20] reported an in-hospital mortality rate of $19.4 \%$ in subjects over 60 years. Both studies did not carry out a nutritional evaluation of the patients. In our study, the malnutrition ratio was not related to hospital stay and slightly to mortality, although the latter association disappeared in the logistic regression model. In the previously mentioned studies, Liu et al. [15] showed a relationship of malnutrition with hospital stay and a worse course of the disease. And finally, Zhang et al. [17] reported in patients with high nutritional risk at ICU admission a higher mortality of ICU 28 days. In our study, albumin, prealbumin, creatinine, and other biochemical parameters showed poor validity when compared with nutritional status by the MUST. These laboratory parameters are influenced by several factors such as inflammation and acute disease [21], and this may explain the lack of association.

Krznaric et al. [3] designed a practical protocol for assessment of nutritional risk without biochemical parameters, including MUST (R-MAPP, as for Remote-Malnutrition APP). This app is an easy and applicable tool for the current situation that we are living with this pandemic. As a first step, MUST should be realized and SARC-F should be performed in older patients [22]. The loss of muscle mass and function (sarcopenia) is an important problem in the older persons and in any patients with an acute or chronic infection disease [22]. Some indirect evidence supports the adverse impact of sarcopenia on COVID-19; for example, patients with sarcopenia had compromised respiratory muscle strength and respiratory function, which were relevant in the treatment of pneumonia [23]. Conversely, COVID-19 could be a risk factor for the incidence of sarcopenia due to the inadequate protein intake and reduced physical activity [24]. Therefore, there is a vicious circle of interaction between COVID-19 and sarcopenia [25]. Recently, Kim et al. [26] have reported that baseline sarcopenia was independently related to prolonged hospital stay in patients with COVID-19. Low pectoralis muscle index, as an indirect parameter of sarcopenia, in patients with COVID-19 was associated with prolonged hospital stay and mortality ratio [27]. Different pathophysiological mechanisms have been impli- cated; for example, angiotensin-converting enzyme 2 is present in muscle tissue which may explain part of the effect of the virus in sarcopenia [28]. Additionally, angiotensin I and II levels have been shown to worsen protein breakdown, increase apoptosis in muscle, and lower muscle regeneration [29].

Some limitations in the present study should be mentioned. First, it is a single-center study in this hospital for treating patients with COVID-19 designed by our government. Second, some other potential risk factors, such as previous physical activity, social support, and lifestyle habits, may also affect the nutritional or muscle status, but the factors were not reported in the present study. Third, we did not perform dynamic nutritional risk assessment, which may provide more information for patients' outcomes. Fourth, the SARC-F questionnaire is a crude measure based on self-reported data, and potential bias could exist. Finally, the absence of the nutritional status of the patients upon discharge from the hospital is a limitation of the study since we cannot show the evolution during admission of the patients who did not die, nor the effect of admission with the treatments administered on the nutritional status, nor if there is any nutritional marker that will improve during admission and could be related to a better or worse prognosis of survival. Despite these limitations, this study has some strengths. This is the first study with this R-MAPP in a real-world design; it could be applicable in other settings for hospitalized or outpatient patients, showing easy use and prediction in mortality.

In conclusion, during COVID-19 infection, hospitalized patients at risk of sarcopenia have a high risk of mortality and have a poor nutritional status. R-MAPP is a quick and easy-to-use tool to evaluate these patients. Possibly, interventions related to sarcopenia could improve the prognosis of patients hospitalized with COVID-19.

\section{Statement of Ethics}

All procedures performed in studies involving human participants were in accordance with the ethical standards of the institutional and/or national research committee and with the 1964 Helsinki Declaration and its later amendments or comparable ethical standards. And, it was approved by our local committee (Hospital Clinic Universitary of Valladolid Committee PI20-1776). Written informed consent was obtained from all individual participants included in the study. 


\section{Conflict of Interest Statement}

The authors have no conflicts of interest to declare.

\section{Funding Sources}

The authors have no funding sources to declare.

\section{Author Contributions}

Daniel Antonio de Luis designed the study and wrote the article. H. Riesgo, S. del Amo, A. Castro, M.J. Sancerferino, Olatz Izaola, J.J. Lopez, and E. Gomez realized nutritional evaluation with R-MAPP. D. Primo realized biochemical evaluation.

\section{Data Availability Statement}

All data generated or analyzed during this study are included in this article. Further enquiries can be directed to the corresponding author.

\section{References}

1 Ralph R, Lew J, Zeng T, Francis M, Xue B, Roux M, et al. 2019- nCoV (Wuhan virus), a novel coronavirus: human-to-human transmission, travel-related cases, and vaccine readiness. J Infect Dev Ctries. 2020;14:3-17.

2 Huang C, Wang Y, Li X, Ren L, Zhao J, Hu Y, et al. Clinical features of patients infected with 2019 novel coronavirus in Wuhan, China. Lancet. 2020;395:497-506.

3 Krznarić Ž, Bender DV, Laviano A, Cuerda C, Landi F, Monteiro R, et al. A simple remote nutritional screening tool and practical guidance for nutritional care in primary practice during the COVID-19 pandemic. Clin Nutr. 2020 Jul;39(7):1983-7.

4 Elia M, chairman, editor. The MUST report: nutritional screening of adults: a multidisciplinary responsibility. Maidenhead, UK: BAPEN (MAG); 2003.

5 Malmstrom TK, Morley JE. Sarcopenia: the target population. J Frailty Aging. 2013;2:55-

6 Stratton RJ, Hackston A, Longmore D, Dixon R, Price S, Stroud M, et al. Malnutrition in hospital outpatients and inpatients: prevalence, concurrent validity and ease of use of the "malnutrition universal screening tool" ("MUST") for adults. Br J Nutr. 2004 Nov; 92(5):799-808

7 Biolo G, Cederholm T, Muscaritoli M. Muscle contractile and metabolic dysfunction is a common feature of sarcopenia of aging and chronic diseases: from sarcopenic obesity to cachexia. Clin Nutr. 2014;33(5):737.

8 Barazzoni R, Bischoff SC, Breda J, Wickramasinghe K, Krznaric Z, Nitzan D, et al. ESPEN expert statements and practical guidance for nutritional management of individuals with SARS-CoV-2 infection. Clin Nutr. 2020 Jun; 39(6):1631-8. Epub 2020 Mar 31.

9 Zheng Z, Peng F, Xu B, Zhao J, Liu H, Peng J, et al. Risk factors of critical \& mortal COVID-19 cases: a systematic literature review and meta-analysis. J Infect. 2020 Aug;81:e1625.

10 Liu K, Chen Y, Lin R, Han K. Clinical features of COVID-19 in elderly patients: a comparison with young and middle-aged patients. J Infect. 2020;80:e14-8.
11 Mehta P, McAuley DF, Brown M, Sanchez E, Tattersall RS, Manson JJ; HLH Across Speciality Collaboration. COVID-19: consider cytokine storm syndromes and immunosuppression. Lancet. 2020;395:1033-4.

12 Zabetakis I, Lordan R, Norton C, Tsoupras A. COVID-19: the inflammation link and the role of nutrition in potential mitigation. $\mathrm{Nu}-$ trients. 2020;12:1466.

13 Handu D, Moloney L, Rozga M, Cheng F. Malnutrition care during the COVID-19 pandemic: considerations for registered dietitian nutritionists. J Acad Nutr Diet. 2021 May;121(5): 979-87.

14 Silva DFO, Lima SCVC, Sena-Evangelista KCM, Marchioni DM, Cobucci RN, Andrade FB. Nutritional risk screening tools for older adults with COVID-19: a systematic review. Nutrients. 2020 Sep 27;12(10):2956.

15 Liu G, Zhang S, Mao Z, Wang W, Hu H. Clinical significance of nutritional risk screening for older adult patients with COVID-19. Eur J Clin Nutr. 2020;74:876-83.

16 Li T, Zhang Y, Gong C, Wang J, Liu B, Shi L, et al. Prevalence of malnutrition and analysis of related factors in elderly patients with COVID-19 in Wuhan, China. Eur J Clin Nutr. 2020;74:871-5.

17 Zhang P, He Z, Yu G, Peng D, Feng Y, Ling J, et al. The modified NUTRIC score can be used for nutritional risk assessment as well as prognosis prediction in critically ill COVID-19 patients. Clin Nutr. 2021 Feb;40(2): 534-41. Epub 2020 Jun 5.

18 Yuan Y, Wang N, Ou X. Caution should be exercised for the detection of SARS-CoV-2, especially in the elderly. J Med Virol. 2020;92: 1641-8.

19 Carrasco-Sánchez FJ, López-Carmona MD, Martínez-Marcos FJ, Pérez-Belmonte LM, Hidalgo-Jiménez A, Buonaiuto V, et al.; SEMICOVID-19 Network. Admission hyperglycaemia as a predictor of mortality in patients hospitalized with COVID-19 regardless of diabetes status: data from the Spanish SEMI-COVID-19 Registry. Ann Med. 2021 Dec;53(1):103-16.

20 Genc Yavuz B, Colak S, Guven R, Altundag I, Seyhan AU, Gunay Inanc R. Clinical features of the 60 years and older patients infected with 2019 novel coronavirus: can we predict mortality earlier? Gerontology. 2021 Mar 30; 67(4):433-40.

21 Hrnciarikova D, Juraskova B, Hyspler R, Solichova D, Ticha A, Klemera P, et al. A changed view of serum prealbumin in the elderly: prealbumin values influenced by concomitant inflammation. Biomed Pap Med Fac Univ Palacky Olomouc Czech Repub. 2007; 151:273-6.

22 Anker SD, Coats AJ, Morley JE, Rosano G, Bernabei R, von Haehling S, et al. Muscle wasting disease: a proposal for a new disease classification. J Cachexia Sarcopenia Muscle. 2014;5(1):1-3

23 Ohara DG, Pegorari MS, Oliveira Dos Santos NL, de Fátima Ribeiro Silva C, Oliveira MSR, Matos AP, et al. Cross-sectional study on the association between pulmonary function and sarcopenia in Brazilian community-dwelling elderly from the Amazon region. J Nutr Health Aging. 2020;24:181-7.

24 Morley JE, Kalantar-Zadeh K, Anker SD. COVID-19: a major cause of cachexia and sarcopenia? J Cachexia Sarcopenia Muscle. 2020 11:863-5.

25 Wang PY, Li Y, Wang Q. Sarcopenia: an underlying treatment target during the $\mathrm{CO}$ VID-19 pandemic. Nutrition. 2021 Apr;84: 111104

26 Kim JW, Yoon JS, Kim EJ, Hong HL, Kwon $\mathrm{HH}$, Jung CY, et al. Prognostic implication of baseline sarcopenia for length of hospital stay and survival in patients with coronavirus disease 2019. J Gerontol A Biol Sci Med Sci. 2021 Jul 13;76(8):e110-6.

27 Ufuk F, Demirci M, Sagtas E, Akbudak IH, Ugurlu E, Sari T. The prognostic value of pneumonia severity score and pectoralis muscle area on chest CT in adult COVID-19 patients. Eur J Radiol. 2020 Oct;131:109271.

28 Kumar NB. Does COVID-19-related cachexia mimic cancer-related cachexia? Examining mechanisms, clinical biomarkers, and potential targets for clinical management. J Cachexia Sarcopenia Muscle. 2021 Apr;12(2):519-22.

29 Bahat G. Covid-19 and the renin angiotensin system: implications for the older adults. J Nutr Health Aging. 2020;24:699-704. 